\title{
Comparison of the toxic effects of different mycotoxins on porcine and mouse oocyte meiosis
}

\author{
Yujie Lu ${ }^{1}$ ， Xiang-Hong Ou ${ }^{2}$, Yue Zhang ${ }^{1}$, Jia-Qian Liu ${ }^{1}$, Peng Zou ${ }^{1}{ }^{\text {, Lu Jia }}{ }^{1}$, Yong-Teng Su ${ }^{3}$, Yu-Rong \\ Sun $^{3}$, Shao-Chen Sun ${ }^{\text {Corresp. } 1}$ \\ ${ }^{1}$ College of Animal Science \& Technology, Nanjing Agricultural University, Nanjing, China \\ 2 Guangdong Second Provincial General Hospital, Guangzhou, China \\ 3 Jiangsu Aomai Bio-tech Company, Nanjing, China \\ Corresponding Author: Shao-Chen Sun \\ Email address: sunsc@njau.edu.cn
}

Background: Aflatoxin B1 (AFB1), Deoxynivalenol (DON), HT-2, ochratoxin A (OTA), Zearalenone (ZEA) are the most common mycotoxins that found in corn-based animal feed which have multiple toxic effects on animals and humans. Previous studies reported that these mycotoxins impaired mammalian oocyte quality. However, the effective concentrations of mycotoxins to animal oocytes were different.

Methods: In this study we aimed to compare the sensitivity of mouse and porcine oocytes to AFB1, DON, HT-2, OTA and ZEA for mycotoxin research. We adopted the polar body extrusion rate of mouse and porcine oocyte as the standard for the effects of mycotoxins on oocyte maturation.

Results and Discussion: Our results showed that $10 \mu \mathrm{M}$ AFB 1 and $1 \mu \mathrm{M}$ DON significantly affected porcine oocyte maturation compared with $50 \mu \mathrm{M}$ AFB1 and $2 \mu \mathrm{M}$ DON on mouse oocytes. However, $10 \mathrm{nM}$ HT-2 significantly affected mouse oocyte maturation compared with 50 nM HT-2 on porcine oocytes. Moreover, $5 \mu \mathrm{M}$ OTA and $10 \mu \mathrm{M}$ ZEA significantly affected porcine oocyte maturation compared with 300 $\mu \mathrm{M}$ OTA and $50 \mu \mathrm{M}$ ZEA on mouse oocytes. In summary, our results showed that porcine oocytes were more sensitive to AFB1, DON, OTA and ZEA than mouse oocytes except HT-2 toxin. 

and mouse oocyte meiosis

3 Yujie Lu${ }^{1}$, Xiang-Hong Ou${ }^{2}$, Yue Zhang ${ }^{1}$, Jia-Qian Liu ${ }^{1}$, Peng Zou ${ }^{1}$, Lu Jia ${ }^{1}$, Yong-Teng Su ${ }^{3}$, Yu-

4 Rong $\operatorname{Sun}^{3}$, Shao-Chen Sun ${ }^{1 *}$

5

$6{ }^{1}$ College of Animal Science and Technology, Nanjing Agricultural University, Nanjing 210095 ,

7 China.

$8 \quad{ }^{2}$ Fertility Preservation Lab, Reproductive Medicine Center, Guangdong Second Provincial

9 General Hospital, Guangzhou, China.

10 3Jiangsu Aomai Bio-tech Company, Nanjing, China.

Running Title: Effects of mycotoxins on oocytes

*Correspondence to: Shao-Chen Sun, College of Animal Sciences and Technology, Nanjing 
24 Abstract

25 Background: Aflatoxin B1 (AFB1), Deoxynivalenol (DON), HT-2, ochratoxin A (OTA),

26 Zearalenone (ZEA) are the most common mycotoxins that found in corn-based animal feed which

27 have multiple toxic effects on animals and humans. Previous studies reported that these mycotoxins impaired mammalian oocyte quality. However, the effective concentrations of mycotoxins to animal oocytes were different.

Methods: In this study we aimed to compare the sensitivity of mouse and porcine oocytes to AFB1, DON, HT-2, OTA and ZEA for mycotoxin research. We adopted the polar body extrusion rate of mouse and porcine oocyte as the standard for the effects of mycotoxins on oocyte maturation.

Results and Discussion: Our results showed that $10 \mu \mathrm{M}$ AFB1 and $1 \mu \mathrm{M}$ DON significantly affected porcine oocyte maturation compared with $50 \mu \mathrm{M}$ AFB1 and $2 \mu \mathrm{M}$ DON on mouse oocytes. However, 10 nM HT-2 significantly affected mouse oocyte maturation compared with 50 nM HT-2 on porcine oocytes. Moreover, $5 \mu \mathrm{M}$ OTA and $10 \mu \mathrm{M}$ ZEA significantly affected porcine oocyte maturation compared with $300 \mu \mathrm{M}$ OTA and $50 \mu \mathrm{M}$ ZEA on mouse oocytes. In summary, our results showed that porcine oocytes were more sensitive to AFB1, DON, OTA and ZEA than mouse oocytes except HT-2 toxin. 


\section{Introduction}

Mycotoxins are secondary metabolites produced by fungi, while the most agriculturally common mycotoxins known today include aflatoxins (AF), Deoxynivalenol (DON), T-2, ochratoxin A (OTA), Zearalenone (ZEA) (Grajewski et al. 2012). These mycotoxins have multiple toxic effects on human and animal health in a very low dose, which draws worldwide attention.

Aflatoxins (AF) are the mycotoxins which widely exist in corn-based animal feed. Considering the toxic potency and carcinogenic action, Aflatoxin B1 is the most important AF (Kew 2013). It causes multiple effects including mitochondrial permeability transition, DNA damage (Shi et al. 2015), oxidative stress (Singh et al. 2015), apoptosis (Peng et al. 2016), the defects of skeletal muscle development in different models (Gunduz \& Oznurlu 2014). In porcine oocytes, AFB1 is shown to affect cell cycle and induce oxidative stress (Liu et al. 2015).

Deoxynivalenol (DON) that produced mainly by fungi is a common contaminant of cereals including maize, wheat and barley (Wu et al. 2011). After feeding the domestic animals and poultry with DON-contaminated feed, it will exist in meat, milk and eggs. In animals, it causes organ damage and hepatic lipid accumulation (Pietsch et al. 2014), emesis, anorexia, growth retardation, immunotoxicity as well as impaired reproduction and development competence (Pestka 2010). At cellular and molecular level, DON can induce apoptosis (Li et al. 2014), oxidative stress and genotoxicity (Yang et al. 2014). In addition, previous study showed that DON affected spindle morphology in porcine oocytes (Han et al. 2016).

HT-2 is the major metabolite of T-2 which is one of the type A trichothecene mycotoxins produced by different Fusarium species. HT-2 causes a myriad of effects including the inhibition 
of protein, DNA and RNA synthesis; oxidative stress (Zhang et al. 2016); reduced reproduction (Zhu et al. 2016) and embryo-fetal toxicity (Wang et al. 2014).

Several fungi including Aspergillus ochraceus, A. carbonarius, A. niger and Penicillium verrucosum produce ochratoxin A (OTA). OTA is toxic to domestic animals, and its main target organ is kidney (Grajewski et al. 2012). OTA causes several effects like immunotoxicity (Al-Anati \& Petzinger 2006), hepatotoxicity, apoptosis, decrease of cell viability, impairment of mouse oocyte maturation and embryonic development (Huang \& Chan 2016).

Zearalenone (ZEA) produced by various Fusarium species is a contaminant of cereal crops and animal feed. Structurally, ZEA is similar to $17 \beta$-estradiol and it will compete with $17 \mathrm{~b}$ estradiol for binding to estrogen receptors, which leads to reproductive disorders (Cortinovis et al. 2013; Minervini et al. 2001; Minervini et al. 2006). The genotoxicity of ZEA has also been confirmed since it induces DNA fragmentation, apoptosis and interruption of cell cycle progression (Abid-Essefi et al. 2003). Several studies showed that ZEA reduced porcine oocyte developmental competence (Hou et al. 2014; Hou et al. 2015; Komsky-Elbaz et al. 2018).

Although previous studies reported that several components of mycotoxins impaired mammalian oocyte quality. However, different effective concentrations were reported in the oocytes of different animal models. Although AFB1, DON, HT-2, OTA and ZEA all have their metabolites in vivo, these mycotoxins are all detected in ovary, indicating that besides their metabolites, AFB1, DON, HT-2, OTA and ZEA could directly affect ovary functions. In this study we aimed to compare the sensitivity of mouse and porcine oocytes to AFB1, DON, HT-2, OTA and ZEA, which could provide the basic data for mycotoxin studies in the future. We adopted the 
87

polar body extrusion rate of mouse and porcine oocyte as the standard for the toxic effects of mycotoxins on oocyte maturation, and our results showed that except HT-2, porcine oocytes were generally more sensitive to AFB1, DON, OTA and ZEA.

\section{Materials and Methods}

\section{Chemicals and regent}

AFB1, DON, HT-2, OTA and ZEA were from J\&K Chemical Ltd. (Shanghai, China). TCM199 was from Gibco (Life Technologies, USA). TCM-199 which contained 75 mg/ml of penicillin, $50 \mathrm{mg} / \mathrm{ml}$ of streptomycin, $0.5 \mathrm{mg} / \mathrm{ml}$ of FSH, $0.5 \mathrm{mg} / \mathrm{ml}$ of LH, $10 \mathrm{ng} / \mathrm{ml}$ of the epidermal growth factor (EGF), and $0.57 \mathrm{mM}$ cysteine was used for oocyte maturation. M2 and M16 culture medium were from Sigma-Aldrich (Merck).

\section{Oocytes collection and culture}

We followed the guidelines of Animal Research Institute Committee of Nanjing Agricultural University to conduct the experiments (SYXK-Su-20170007). Germinal vesicle-intact oocytes of mice that obtained from ovaries of 3- to 5-week old ICR mice were collected in M2 medium and cultured with M16 medium (Sigma Chemical Co., St. Louis, MO) under the paraffin oil. These oocytes of mice were placed at $37^{\circ} \mathrm{C}$ with $5 \% \mathrm{CO}_{2}$ for 12 hours to observe the polar body extrusion. Ovaries of Duroc pigs were collected from prepubertal gilts purchased at a local slaughterhouse of Feng Yong Food Industry. After slaughter, ovaries were placed in a thermos bottle which contained $0.9 \%$ physiological saline and then delivered to our laboratory within 2 hours. The temperature of the thermos bottle was close to $38^{\circ} \mathrm{C}$. Once the ovaries were delivered, they were 
washed with sterile saline. We aspirated follicular fluids from 3 to $6 \mathrm{~mm}$ antral follicles with a 10 $\mathrm{ml}$ disposable syringe and an $18 \mathrm{G}$ needle. Cumulus oocyte complexes $\left(\mathrm{COC}_{\mathrm{S}}\right)$ with intact and compact cumulus were selected for maturation. These oocytes were placed at $38.5^{\circ} \mathrm{C}$ with $5 \% \mathrm{CO}_{2}$ for 44 hours to observe the polar body extrusion.

\section{Toxin treatment}

AFB1, DON, HT-2, OTA and ZEA were dissolved and stored at $50 \mathrm{mM}$ in DMSO. and then diluted into different concentrations with M199 or M16 maturation medium. The GV oocytes were then cultured with these mycotoxins to analyze the maturation rate by the polar body extrusion index. The same quantity of DMSO was added in the control group.

\section{Statistical analysis}

Data are presented as means $(n=3)$. The concentration-response curves were made by GraphPad Prism 5. At least three biological replicates were used for each analysis. Each replicate was done by an independent experiment at the different time. Results are given as means \pm SEM, and two groups were compared by student t-test. A p-value of $<0.05$ was considered significant.

\section{Results}

\section{Effects of AFB1 on mouse and porcine oocyte maturation}

We first examined the effects of AFB1 on mouse and porcine oocytes. Mouse oocytes were cultured for 12 hours with 10,50,100 and $150 \mu \mathrm{M}$ AFB1. The average polar body extrusion rate of the control group was $79.94 \pm 4.3 \%(n=142)$ (Figure 1A). Compared with control oocytes, when oocytes were cultured with $10 \mu \mathrm{M}$ AFB1, rate of polar body extrusion showed no significant 
129

130

131

132

133

134

135

136

137

138

139

140

141

142

143

144

145

146

147

148

149

difference $(72.82 \pm 4.83 \%, \mathrm{n}=110, \mathrm{p}>0.05)$. Rates of matured oocytes were significantly decreased

when the concentration of AFB1 were increased to $50 \mu \mathrm{M}(55.96 \pm 4.77 \%, \mathrm{n}=157, \mathrm{p}<0.05), 100$

$\mu \mathrm{M}(17.86 \pm 7.51 \%, \mathrm{n}=154, \mathrm{p}<0.05), 150 \mu \mathrm{M}(2.15 \pm 3.72 \%, \mathrm{n}=146, \mathrm{p}<0.001)$. We cultured the

porcine oocytes for $44 \mathrm{~h}$ with $5,10,50,100 \mu \mathrm{M}$ AFB1. The polar body extrusion rate was $80.72 \pm 7.05 \%(n=217)$ in the control group of porcine oocytes, which was close to mouse oocytes

However, the rate of polar body extrusion in porcine oocytes was decreased with the increased

AFB1 concentration. Porcine oocytes cultured with $5 \mu \mathrm{M}$ AFB1 and the control oocytes showed no significant difference $(68.34 \pm 15.39 \%, n=195$ vs $80.72 \pm 7.05 \% n=217, p>0.05)$. However, the rate of polar body extrusion in porcine oocytes was significantly decreased with $10 \mu \mathrm{M}$ (53.02 $\pm 7.12 \%, \mathrm{n}=144, \mathrm{p}<0.001), 50 \mu \mathrm{M}(22.95 \pm 6.76 \%, \mathrm{n}=192, \mathrm{p}<0.01), 100 \mu \mathrm{M}(0 \pm 0 \%, \mathrm{n}=147$ $\mathrm{p}<0.01)$ AFB1 treatment (Figure 1B). We also analyzed the mouse groups and porcine groups at the same concentrations. With the same concentration of AFB1 treatment, the rate of porcine oocyte polar body extrusion was lower than mouse oocytes. As shown in Figure 1C, the rates of the mouse and porcine control groups showed no significant difference $(p>0.05)$, while there were significant differences when the concentrations were $10 \mu \mathrm{M}(\mathrm{p}<0.05)$ and $50 \mu \mathrm{M}(\mathrm{p}<0.01)$, indicating that compared with mouse oocytes, porcine oocytes were more sensitive to AFB1.

\section{Effects of DON on mouse and porcine oocyte maturation}

We next examined the effects of DON on mouse and porcine oocytes. Mouse oocytes were cultured for 12 hours with 1, 2, 3 and $4 \mu \mathrm{M}$ DON. Our results showed that DON affected mouse oocyte maturation. The average polar body extrusion rate of the control group was $76.73 \pm 3.24 \%$ $(\mathrm{n}=162)$ (Figure 2A), when mouse oocytes cultured with $1 \mu \mathrm{M}$ AFB1, there was no significantly 
150

151

152

153

154

155

156

157

158

159

160

161

162

163

164

165

166

167

168

169

170

difference between the control group and $1 \mu \mathrm{M}$ group $(76.01 \pm 2.35 \% \mathrm{n}=139, \mathrm{p}>0.05)$. While the rate of polar body extrusion in mouse oocytes was significantly decreased with $2 \mu \mathrm{M}$ DON treatment $(44.38 \pm 4.87 \%, \mathrm{n}=173, \mathrm{p}<0.05), 3 \mu \mathrm{M}$ DON treatment $(16.30 \pm 4.00 \%, \mathrm{n}=199, \mathrm{p}<0.001)$, $4 \mu$ M DON treatment $(2.28 \pm 0.69 \%, \mathrm{n}=189, \mathrm{p}<0.001)$, compared with the control group. We cultured the porcine oocytes for $44 \mathrm{~h}$ with $0.5,1,2,3 \mu \mathrm{M}$ DON. The polar body extrusion rate was $72.05 \pm 2.6 \%(n=195)$ in the control group of porcine oocytes, which was close to mouse oocytes.

At the concentration of $0.5 \mu \mathrm{M}$, the control group and $0.5 \mu \mathrm{M}$ treatment group $(68.42 \pm 4.55 \%$ $\mathrm{n}=159, \mathrm{p}>0.05)$ showed no significant difference. However, the rate of polar body extrusion in porcine oocytes was significantly decreased with $1 \mu \mathrm{M}$ DON treatment $(46.29 \pm 3.89 \%, \mathrm{n}=176$, $\mathrm{p}<0.05), 2 \mu \mathrm{M}$ DON treatment $(17.02 \pm 4.87 \%, \mathrm{n}=145, \mathrm{p}<0.01), 3 \mu \mathrm{M}$ DON treatment $(3.29 \pm 1.81 \%, \mathrm{n}=132, \mathrm{p}<0.001)$ (Figure $2 \mathrm{~B})$. To compare the sensitivity of mouse and porcine, we also analyze the rate of same concentrations. The rates of control groups were close to each other, while at the concentrations of $1 \mu \mathrm{M}(\mathrm{p}<0.001), 2 \mu \mathrm{M}(\mathrm{p}<0.01), 3 \mu \mathrm{M}(\mathrm{p}<0.01)$, polar body extrusion rates of mouse oocytes and porcine oocytes showed significant difference (Figure 2C), indicating that compared with mouse oocytes, porcine oocytes were more sensitive to DON.

\section{Effects of HT-2 on mouse and porcine oocyte maturation}

We next examined the effects of HT-2 on mouse and porcine oocytes. The mouse oocytes were cultured for 12 hours with 10, 20,30 and $40 \mathrm{nM}$ HT-2. The average polar body extrusion rate of the control group was $73.08 \pm 1.67 \%(n=153)$ (Figure $3 \mathrm{~A})$, while the rate of polar body extrusion in mouse oocytes was significantly decreased with $10 \mathrm{nM}$ HT-2 treatment $(43.33 \pm 4.93 \%, \mathrm{n}=176$, $\mathrm{p}<0.05), 20 \mathrm{nM}$ HT-2 treatment $(33.05 \pm 2.18 \%, \mathrm{n}=163, \mathrm{p}<0.001), 30 \mathrm{nM}$ HT-2 treatment 
$171(7.08 \pm 0.89 \%, \mathrm{n}=150, \mathrm{p}<0.001), 40 \mathrm{nM}$ HT-2 treatment $(1.05 \pm 0.10 \%, \mathrm{n}=169, \mathrm{p}<0.01)$. The porcine

172 oocytes were cultured for $44 \mathrm{~h}$ with 10, 50, 100, 150, 200 and $400 \mathrm{nM}$ HT-2. Similar with the

173 mouse oocytes, our results showed that HT-2 affected porcine oocyte maturation. The polar body

174 extrusion rate was $78.19 \pm 2.03 \%(n=171)$ in the control group of porcine oocytes, which was close

175 to mouse oocytes. However, except the 10 nM HT-2 treatment $(68.67 \pm 2.99 \%, n=163, p>0.05)$, the

176 rate of polar body extrusion in porcine oocytes was significantly decreased with 50 nM HT-2

177 treatment $(47.4 \pm 3.36 \%, \mathrm{n}=156, \mathrm{p}<0.01), 100 \mathrm{nM} \mathrm{HT}-2$ treatment $(25.50 \pm 5.14 \%, \mathrm{n}=164, \mathrm{p}<0.01)$,

$178150 \mathrm{nM}$ HT-2 treatment $(21.22 \pm 4.07 \%, \mathrm{n}=188, \mathrm{p}<0.01), 200 \mathrm{nM}$ HT-2 treatment $(18.02 \pm 6.69 \%$,

$179 \mathrm{n}=175, \mathrm{p}<0.01), 400 \mathrm{nM}$ HT-2 treatment $(8.22 \pm 0.78 \%, \mathrm{n}=162, \mathrm{p}<0.001)$ (Figure $3 \mathrm{~B})$. The control

180 groups in mouse and porcine have similar values, however, at the concentration of $10 \mu \mathrm{M}(\mathrm{p}<0.01)$

181 rates of mouse and porcine were significantly different (Figure 3C). Our results showed that with

182 the same concentration of HT-2 treatment the rate of mouse oocyte polar body extrusion was lower

than porcine oocytes, indicating that compared with porcine oocytes mouse oocytes were more

sensitive to HT-2.

185

186

\section{Effects of OTA on mouse and porcine oocyte maturation}

We next examined the effects of OTA on mouse and porcine oocytes. Mouse oocytes were cultured for 12 hours with 200, 300, 400 and $600 \mu \mathrm{M}$ OTA. The average polar body extrusion rate of the control group was $80.23 \pm 3.87 \%(n=169)$ (Figure $4 A)$, compared with the control group, the concentration of $200 \mu \mathrm{M}$ OTA showed no significant difference $(73.77 \pm 2.24 \% \mathrm{n}=143, \mathrm{p}>0.05)$, while the rate of polar body extrusion in mouse oocytes was significantly decreased with $300 \mu \mathrm{M}$ OTA treatment $(49.86 \pm 4.29 \%, \mathrm{n}=190, \mathrm{p}<0.01), 400 \mu$ M OTA treatment $(31.23 \pm 3.64 \%, \mathrm{n}=145$, 
$192 \mathrm{p}<0.01), 600 \mu \mathrm{M}$ OTA treatment $(2.22 \pm 3.85 \%, \mathrm{n}=178, \mathrm{p}<0.01)$. The porcine oocytes were cultured

193 for $44 \mathrm{~h}$ with $1,5,10,30$ and $100 \mu$ M OTA. Similar with the mouse oocytes, our results showed

194 that OTA affected porcine oocyte maturation. The control group and $1 \mu \mathrm{M}$ OTA group showed no

195 significant difference $(80.34 \pm 7.95 \% n=164$ vs $65.46 \pm 4.09 \% n=178, p>0.05)$. However, the rate of

196 polar body extrusion in porcine oocytes was significantly decreased with $5 \mu$ M OTA treatment

$197(53.83 \pm 0.34 \%, \mathrm{n}=154, \mathrm{p}<0.05), 10 \mu \mathrm{M}$ OTA treatment $(22.26 \pm 3.14 \%, \mathrm{n}=183, \mathrm{p}<0.05), 30 \mu \mathrm{M}$

OTA treatment $(22.19 \pm 4.87 \%, \mathrm{n}=140, \mathrm{p}<0.01), 100 \mu \mathrm{M}$ OTA treatment $(4.24 \pm 3.93 \%, \mathrm{n}=144$,

$\mathrm{p}<0.01$ ) (Figure 4B). With the same concentration of OTA treatment, the rate of porcine oocyte

polar body extrusion was lower than mouse oocytes (Figure 4C), indicating that compared with

mouse oocytes, porcine oocytes were more sensitive to OTA.

\section{Effects of ZEA on mouse and porcine oocyte maturation}

The last we examined was the effects of ZEA on mouse and porcine oocytes. Mouse oocytes

were cultured for 12 hours with 10, 50, 100 and $200 \mu \mathrm{M}$ ZEA. The average polar body extrusion

rate of the control group was $81.29 \pm 6.06 \%(n=155)$ (Figure $5 A)$, when the concentration was 10

$\mu \mathrm{M}$, the average rate of MII oocytes was $74.52 \pm 4.92 \%(n=154)$ which showed no significant

difference with the control groups $(\mathrm{p}>0.05)$. While the rate of polar body extrusion in mouse

oocytes was significantly decreased with $50 \mu \mathrm{M}$ ZEA treatment $(54.35 \pm 3.9 \%, \mathrm{n}=128, \mathrm{p}<0.05)$,

$100 \mu \mathrm{M}$ ZEA treatment $(26.23 \pm 8.00 \%, \mathrm{n}=150, \mathrm{p}<0.05), 200 \mu \mathrm{M}$ ZEA treatment $(0.00 \pm 0.00 \%$,

$\mathrm{n}=132, \mathrm{p}<0.01$ ). For porcine oocytes, the average polar body extrusion rate of the control groups

was $77.85 \pm 9.51 \%(n=175)$, while the $5 \mu$ M ZEA groups $(60.45 \pm 1.65 \%, n=199, p>0.05)$ showed

no significant differences with the control groups. However, the rate of polar body extrusion in 
213 porcine oocytes was significantly decreased with the $10 \mu \mathrm{M}$ groups $(51.42 \pm 2.73 \%, \mathrm{n}=190$,

$214 \mathrm{p}<0.05), 30 \mu \mathrm{dM}$ ZEA treatment $(19.10 \pm 4.49 \%, \mathrm{n}=147, \mathrm{p}<0.05)$ and $50 \mu \mathrm{M}$ ZEA treatment

$215(4.08 \pm 4.08 \%, \mathrm{n}=148, \mathrm{p}<0.01)$ (Figure 5B). Our results showed that with the same concentration

216 of ZEA treatment, the rate of porcine oocyte polar body extrusion was lower than mouse oocytes.

217 Control groups of mouse and porcine oocytes showed no significant difference $(\mathrm{p}>0.05)$. However,

218 there were significant difference between mouse oocytes and porcine oocytes at $10 \mu \mathrm{M}(\mathrm{p}<0.01)$

219 and $50 \mu \mathrm{M}(\mathrm{p}<0.001)$ (Figure 5C), indicating that compared with mouse oocytes, porcine oocytes 220 were more sensitive to ZEA.

\section{Discussion}

In present study we used the polar body extrusion as the index for oocyte maturation to compare the sensitivity of mouse and porcine oocytes to AFB1, DON, HT-2, OTA and ZEA. And our results provided the basic database for the mycotoxin on oocyte studies.

Mycotoxins are shown to affect human and animal health from multiple aspects, such as immune system, micro-organisms. And recent years, the toxicity of mycotoxins on reproductive system especially on oocytes and sperms were reported. Our previous work found that when 50 $\mu \mathrm{M}$ AFB1 affected cumulus oocyte complexes growth, especially the polar body extrusion was significantly reduced in porcine oocytes (Liu et al. 2015). However, $10 \mu \mathrm{M}$ AFB1 significantly increased the proportion of sperm with fragmented DNA in mice (Komsky-Elbaz et al. 2017). This indicated that even in the reproductive system, the sensitivity of different cell types or animal 
234

235

236

237

238

239

240

241

242

243

244

245

246

247

248

249

250

251

252

253

254

oocyte maturation instead of $50 \mu \mathrm{M}$ AFB1 in mouse oocytes.

$2 \mu \mathrm{M}$ DON was shown to effect the formation of the meiotic spindle in porcine oocytes (Hou et al. 2015). While a recent study showed that $10 \mu \mathrm{M}$ DON affected the morphology of pig ovaries with an ex vivo approach (Gerez et al. 2017). Our recent study also showed that $3 \mu \mathrm{M}$ DON exposure altered autophagy/apoptosis and epigenetic modifications in porcine oocytes (Han et al. 2016). In present study our results showed that $1 \mu \mathrm{M}$ DON affected porcine oocyte maturation instead of $2 \mu \mathrm{M}$ DON in mouse oocytes, which showed similar sensitivity pattern to AFB1. However, HT-2 had different sensitivity pattern compared with AFB1 and DON. HT-2 toxin was shown to affect cytoskeletal dynamics, apoptosis/autophagy, oxidative stress, and epigenetic modifications in mouse oocytes (Zhu et al. 2016). Our results showed that $10 \mathrm{nM} \mathrm{HT}-2$ affected mouse oocyte maturation while the similar results only occurred at $50 \mathrm{nM} \mathrm{HT}-2$ exposure for porcine oocytes. Further study is still needed to explore the toxic effects of HT-2 toxin in different reproductive cell types like cumulus cells and sperm.

Recent study indicated that OTA significantly impaired oocyte maturation, IVF rates and inhibited embryonic development in vitro, because OTA could induce caspase-dependent apoptosis with in vivo model (Huang \& Chan 2016). 1-10 $\mu \mathrm{M}$ OTA in the drinking water was adopted in this study. Our results showed that $10 \mu \mathrm{M}$ OTA affected porcine oocyte maturation instead of $300 \mu \mathrm{M}$ OTA in mouse oocytes. The big difference for the OTA in mouse oocyte between in vivo and in vitro model needs more study to explain. For ZEA, at the concentration of $30 \mu \mathrm{M}$, ZEA was shown to affect porcine oocyte maturation and embryonic development through oxidative stress, autophagy and early apoptosis (Komsky-Elbaz et al. 2018); and for mouse 
255

256

257

258

259

260

261

262

263

264

265

266

267

268

269

270

271

272

273

274

275

276

277

278

279

280

281

282

oocytes, it affected oocyte quality by altering the epigenetic modification levels (Zhu et al. 2014).

Our results showed that $10 \mu \mathrm{M}$ ZEA affected porcine oocyte maturation instead of $50 \mu \mathrm{M} Z \mathrm{ZEA}$ in mouse oocytes, which showed similar concentration pattern with AFB1 and DON.

\section{Conclusion}

In all, our results showed that these five mycotoxins all affected mouse and porcine oocyte quality, however, different sensitivity pattern between mouse and porcine oocytes were found. Generally porcine oocytes were more sensitive to AFB1, DON, OTA, ZEA compared with mouse oocytes except HT-2. Our results provided basic database for the further studies on mammalian oocytes.

\section{Availability of data}

All data generated or analyzed in this study are included in this published article.

\section{References}

Abid-Essefi S, Baudrimont I, Hassen W, Ouanes Z, Mobio TA, Anane R, Creppy EE, and Bacha H. 2003. DNA fragmentation, apoptosis and cell cycle arrest induced by zearalenone in cultured DOK, Vero and Caco-2 cells: prevention by Vitamin E. Toxicology 192:237-248. 10.1016/s0300-483x(03)00329-9

Al-Anati L, and Petzinger E. 2006. Immunotoxic activity of ochratoxin A. J Vet Pharmacol Ther 29:79-90. 10.1111/j.1365-2885.2006.00718.x

Cortinovis C, Pizzo F, Spicer LJ, and Caloni F. 2013. Fusarium mycotoxins: effects on reproductive function in domestic animals--a review. Theriogenology 80:557-564. 10.1016/j.theriogenology.2013.06.018

Gerez JR, Desto SS, and Bracarense A. 2017. Deoxynivalenol induces toxic effects in the ovaries of pigs: An ex vivo approach. Theriogenology 90:94-100. 10.1016/j.theriogenology.2016.10.023

Grajewski J, Blajet-Kosicka A, Twaruzek M, and Kosicki R. 2012. Occurrence of mycotoxins in Polish animal feed in years 2006-2009. J Anim Physiol Anim Nutr (Berl) 96:870-877. 10.1111/j.1439-0396.2012.01280.x

Gunduz N, and Oznurlu Y. 2014. Adverse effects of aflatoxin B1 on skeletal muscle development in broiler chickens. Br Poult Sci 55:684-692. 10.1080/00071668.2014.949621

Han J, Wang QC, Zhu CC, Liu J, Zhang Y, Cui XS, Kim NH, and Sun SC. 2016. Deoxynivalenol exposure induces autophagy/apoptosis and epigenetic modification changes during porcine oocyte maturation. Toxicol Appl 
Pharmacol 300:70-76. 10.1016/j.taap.2016.03.006

Hou YJ, Xiong B, Zheng WJ, Duan X, Cui XS, Kim NH, Wang Q, Xu YX, and Sun SC. 2014. Oocyte quality in mice is affected by a mycotoxin-contaminated diet. Environ Mol Mutagen 55:354-362. 10.1002/em.21833

Hou YJ, Zhu CC, Xu YX, Cui XS, Kim NH, and Sun SC. 2015. Zearalenone exposure affects mouse oocyte meiotic maturation and granulosa cell proliferation. Environ Toxicol 30:1226-1233. 10.1002/tox.21995

Huang FJ, and Chan WH. 2016. Effects of ochratoxin a on mouse oocyte maturation and fertilization, and apoptosis during fetal development. Environ Toxicol 31:724-735. 10.1002/tox.22085

Kew MC. 2013. Aflatoxins as a cause of hepatocellular carcinoma. J Gastrointestin Liver Dis 22:305-310.

Komsky-Elbaz A, Saktsier M, and Roth Z. 2017. Aflatoxin B1 impairs sperm quality and fertilization competence. Toxicology 393:42-50. 10.1016/j.tox.2017.11.007

Komsky-Elbaz A, Saktsier M, and Roth Z. 2018. Aflatoxin B1 impairs sperm quality and fertilization competence. Toxicology 393:42-50. 10.1016/j.tox.2017.11.007

Li D, Ma H, Ye Y, Ji C, Tang X, Ouyang D, Chen J, Li Y, and Ma Y. 2014. Deoxynivalenol induces apoptosis in mouse thymic epithelial cells through mitochondria-mediated pathway. Environ Toxicol Pharmacol 38:163171. 10.1016/j.etap.2014.05.015

Liu J, Wang QC, Han J, Xiong B, and Sun SC. 2015. Aflatoxin B1 is toxic to porcine oocyte maturation. Mutagenesis 30:527-535. 10.1093/mutage/gev015

Minervini F, Dell'Aquila ME, Maritato F, Minoia P, and Visconti A. 2001. Toxic effects of the mycotoxin zearalenone and its derivatives on in vitro maturation of bovine oocytes and 17 beta-estradiol levels in mural granulosa cell cultures. Toxicol In Vitro 15:489-495.

Minervini F, Giannoccaro A, Fornelli F, Dell'Aquila ME, Minoia P, and Visconti A. 2006. Influence of mycotoxin zearalenone and its derivatives (alpha and beta zearalenol) on apoptosis and proliferation of cultured granulosa cells from equine ovaries. Reprod Biol Endocrinol 4:62. 10.1186/1477-7827-4-62

Peng X, Chen K, Chen J, Fang J, Cui H, Zuo Z, Deng J, Chen Z, Geng Y, and Lai W. 2016. Aflatoxin B1 affects apoptosis and expression of Bax, Bcl-2, and Caspase- 3 in thymus and bursa of fabricius in broiler chickens. Environ Toxicol 31:1113-1120. 10.1002/tox.22120

Pestka JJ. 2010. Deoxynivalenol: mechanisms of action, human exposure, and toxicological relevance. Arch Toxicol 84:663-679. 10.1007/s00204-010-0579-8

Pietsch C, Schulz C, Rovira P, Kloas W, and Burkhardt-Holm P. 2014. Organ damage and hepatic lipid accumulation in carp (Cyprinus carpio L.) after feed-borne exposure to the mycotoxin, deoxynivalenol (DON). Toxins (Basel) 6:756-778. 10.3390/toxins6020756

Shi D, Liao S, Guo S, Li H, Yang M, and Tang Z. 2015. Protective effects of selenium on aflatoxin B1-induced mitochondrial permeability transition, DNA damage, and histological alterations in duckling liver. Biol Trace Elem Res 163:162-168. 10.1007/s12011-014-0189-z

Singh KB, Maurya BK, and Trigun SK. 2015. Activation of oxidative stress and inflammatory factors could account for histopathological progression of aflatoxin-B1 induced hepatocarcinogenesis in rat. Mol Cell Biochem 401:185-196. 10.1007/s11010-014-2306-x

Wang X, Wang W, Cheng G, Huang L, Chen D, Tao Y, Pan Y, Hao H, Wu Q, Wan D, Liu Z, Wang Y, and Yuan Z. 2014. High risk of embryo-fetal toxicity: placental transfer of T-2 toxin and its major metabolite HT-2 toxin in BeWo cells. Toxicol Sci 137:168-178. 10.1093/toxsci/kft233

Wu Q, Lohrey L, Cramer B, Yuan Z, and Humpf HU. 2011. Impact of physicochemical parameters on the 
decomposition of deoxynivalenol during extrusion cooking of wheat grits. J Agric Food Chem 59:1248012485. 10.1021/jf2038604

Yang W, Yu M, Fu J, Bao W, Wang D, Hao L, Yao P, Nussler AK, Yan H, and Liu L. 2014. Deoxynivalenol induced oxidative stress and genotoxicity in human peripheral blood lymphocytes. Food Chem Toxicol 64:383-396. 10.1016/j.fct.2013.12.012

Zhang Y, Han J, Zhu CC, Tang F, Cui XS, Kim NH, and Sun SC. 2016. Exposure to HT-2 toxin causes oxidative stress induced apoptosis/autophagy in porcine oocytes. Sci Rep 6:33904. 10.1038/srep33904

Zhu CC, Hou YJ, Han J, Cui XS, Kim NH, and Sun SC. 2014. Zearalenone exposure affects epigenetic modifications of mouse eggs. Mutagenesis 29:489-495. 10.1093/mutage/geu033

Zhu CC, Zhang Y, Duan X, Han J, and Sun SC. 2016. Toxic effects of HT-2 toxin on mouse oocytes and its possible mechanisms. Arch Toxicol 90:1495-1505. 10.1007/s00204-015-1560-3

\section{Figure legend}

Figure 1 The effects of AFB1 on the oocyte maturation. (A) Rate of polar body extrusion after AFB1 treatment. Mouse oocytes were cultured with 0, 10, 50, 100, $150 \mu \mathrm{M}$ AFB1 for $12 \mathrm{~h}$. (B) Rate of polar body extrusion after AFB1 treatment. Porcine oocytes were cultured with 0, 5, 10, 50, $100 \mu \mathrm{M}$ AFB1 for $44 \mathrm{~h}$. Both polar body extrusion rates of mouse and porcine oocytes were reduced with the increasing concentration. (C) The statistical analysis for the effects of same concentration on mouse and porcine oocytes. The grey line represented rate of mouse polar body extrusion. The black line represented rate of porcine polar body extrusion. ${ }^{*}$, significance, $\mathrm{p}<0.05$; **, significance, $\mathrm{p}<0.01 ; * * *$, significance, $\mathrm{p}<0.001$.

Figure 2 The effects of DON on the oocyte maturation. (A) Rate of polar body extrusion after DON treatment. For mouse oocyte culture, the DON concentration was $0,1,2,3,4 \mu \mathrm{M}$. (B) Rate of polar body extrusion after DON treatment. For porcine oocytes, DON concentration was $0,0.5$, 1, 2, $3 \mu \mathrm{M}$. The reduction of polar body extrusion was does-dependent. (C) The statistical analysis for the effects of same concentration on mouse and porcine oocytes. The grey line represented rate 
351 of mouse polar body extrusion. The black line represented rate of porcine polar body extrusion. *,

352 significance, $\mathrm{p}<0.05 ; * *$, significance, $\mathrm{p}<0.01$; ***, significance, $\mathrm{p}<0.001$.

353 Figure 3 The effects of HT-2 on the oocyte maturation. (A) Rate of polar body extrusion after HT-

3542 treatment. Mouse oocytes were cultured with HT-2 at the concentration of 0, 10, 20, 30, $40 \mathrm{nM}$.

355 (B) Rate of polar body extrusion after HT-2 treatment. For porcine oocytes culture, HT-2 was 0,

$10,50,100,150,200,400 \mathrm{nM}$. The rate of polar body extrusion was decreased when the

concentration of HT-2 was increasing. (C) The statistical analysis for the effects of same

concentration on mouse and porcine oocytes. The grey line represented rate of mouse polar body

extrusion. The black line represented rate of porcine polar body extrusion. ${ }^{*}$, significance, $\mathrm{p}<0.05$;

360

**, significance, $\mathrm{p}<0.01 ; * * *$, significance, $\mathrm{p}<0.001$.

Figure 4 The effects of OTA on the oocyte maturation. (A) Rate of polar body extrusion after

OTA treatment. For mouse oocyte culture, OTA concentration was 0, 200, 300, 400, $600 \mu \mathrm{M}$. (B)

Rate of polar body extrusion after OTA treatment. For porcine oocyte culture, OTA concentration

was $0,1,5,10,30,100 \mu \mathrm{M}$. *, significance, $\mathrm{p}<0.05$; **, significance, $\mathrm{p}<0.01$. (C) The comparison

for the effects of different concentration on mouse and porcine oocytes. The grey line represented rate of mouse polar body extrusion. The black line represented rate of porcine polar body extrusion.

Figure 5 The effects of ZEA on the oocyte maturation. (A) Rate of polar body extrusion after ZEA

treatment. Mouse oocytes were cultured with 0, 10, 50, 100, $200 \mu \mathrm{M}$ ZEA. (B) Rate of polar body

extrusion after ZEA treatment. Porcine oocytes were cultured with 0, 5, 10, 30, $50 \mu \mathrm{M}$ ZEA. Both

mouse and porcine polar body extrusion rate were reducing when ZEA concentration was 
372 oocytes. The grey line represented rate of mouse polar body extrusion. The black line represented

373 rate of porcine polar body extrusion. *, significance, $\mathrm{p}<0.05$; **, significance, $\mathrm{p}<0.01$. 


\section{Figure 1}

The effects of AFB1 on the oocyte maturation

(A) Rate of polar body extrusion after AFB1 treatment. Mouse oocytes were cultured with 0 , 10, 50, 100, $150 \mu \mathrm{M}$ AFB1 for $12 \mathrm{~h}$. (B) Rate of polar body extrusion after AFB1 treatment. Porcine oocytes were cultured with 0, 5, 10, 50, $100 \mu \mathrm{M}$ AFB1 for $44 \mathrm{~h}$. Both polar body extrusion rates of mouse and porcine oocytes were reduced with the increasing concentration. (C) The statistical analysis for the effects of same concentration on mouse and porcine oocytes. The grey line represented rate of mouse polar body extrusion. The black line represented rate of porcine polar body extrusion. $*$, significance, $\mathrm{p}<0.05$; **, significance, $p<0.01 ; * * *$, significance, $p<0.001$. 
A

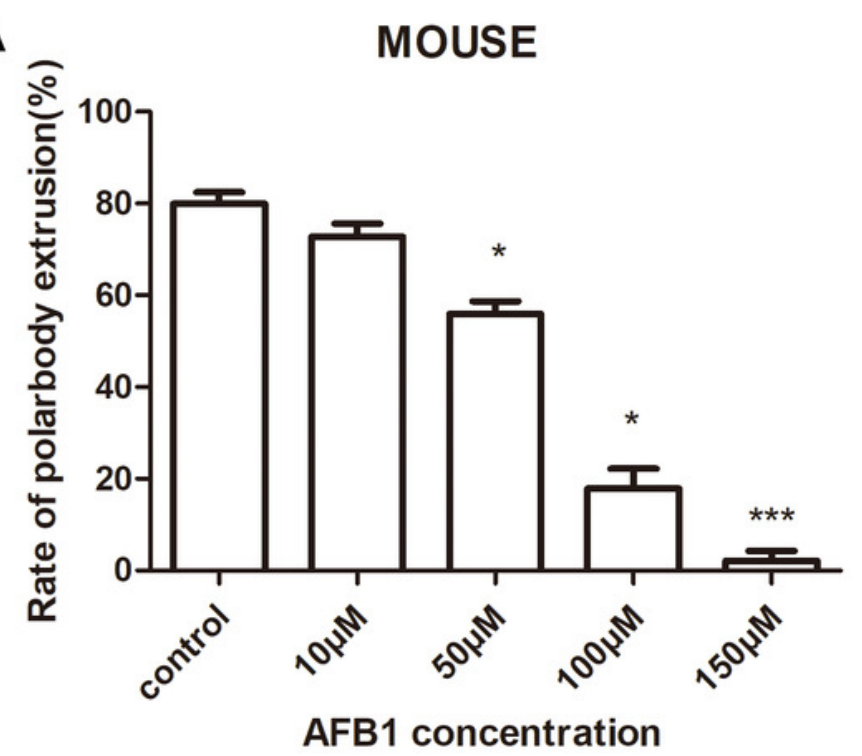

C

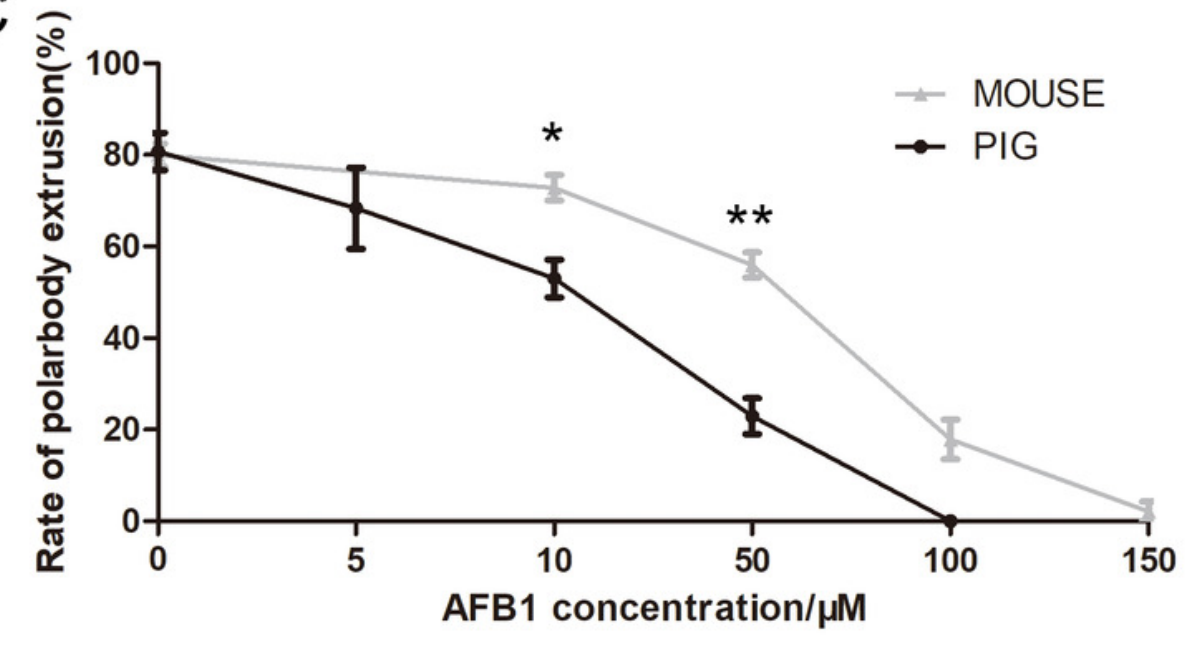

B

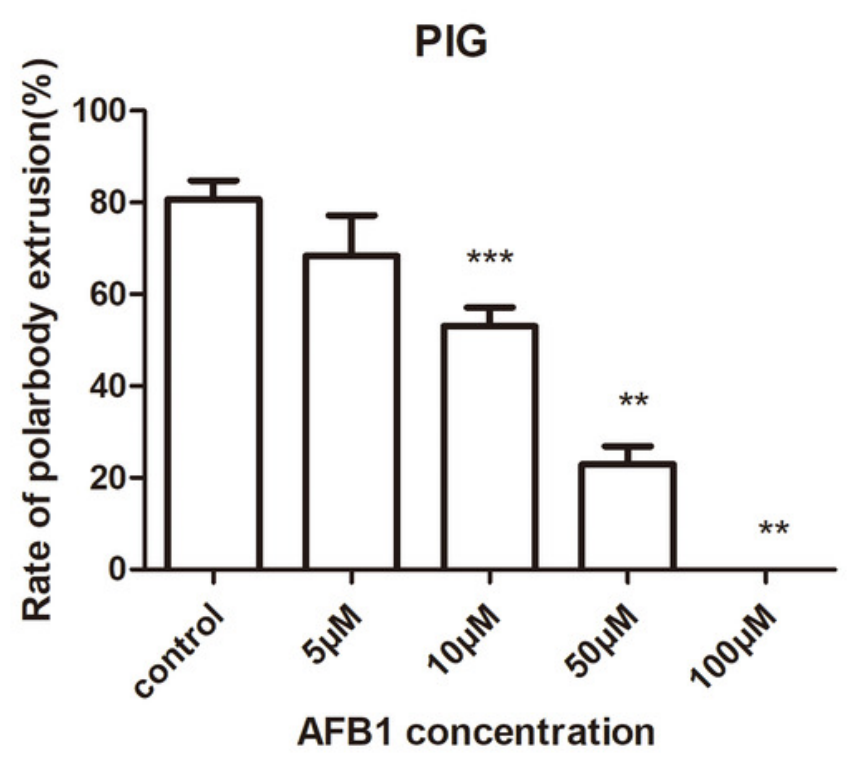


Figure 2

The effects of DON on the oocyte maturation

(A) Rate of polar body extrusion after DON treatment. For mouse oocyte culture, the DON concentration was $0,1,2,3,4 \mu \mathrm{M}$. (B) Rate of polar body extrusion after DON treatment. For porcine oocytes, DON concentration was $0,0.5,1,2,3 \mu \mathrm{M}$. The reduction of polar body extrusion was does-dependent. (C) The statistical analysis for the effects of same concentration on mouse and porcine oocytes. The grey line represented rate of mouse polar body extrusion. The black line represented rate of porcine polar body extrusion. *, significance, $p<0.05 ; * *$, significance, $p<0.01 ; * * *$, significance, $p<0.001$.

A

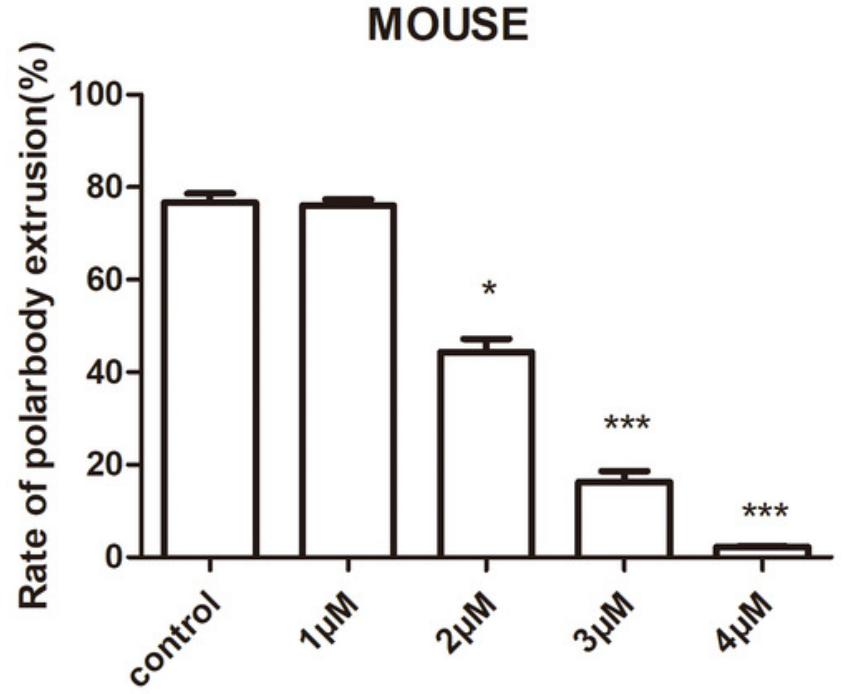

DON concentration

C

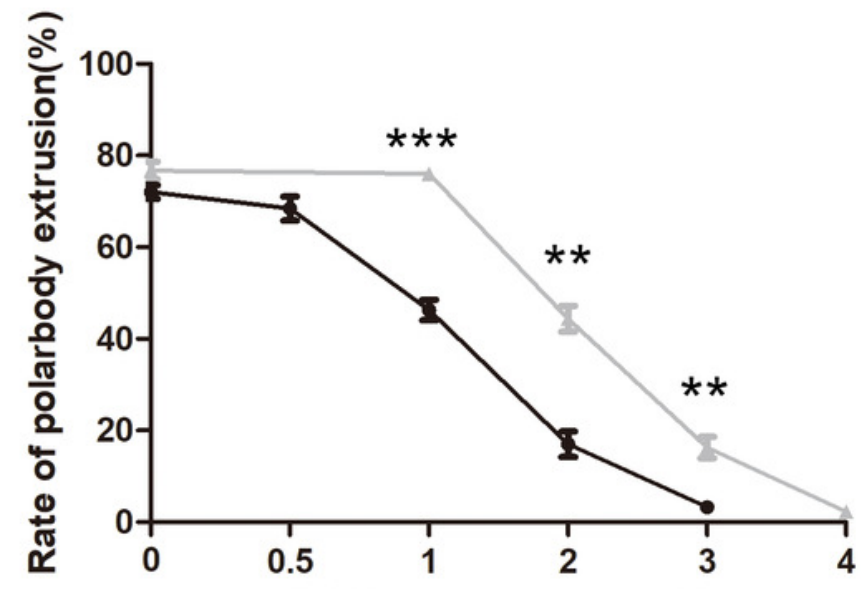

DON concentration/uM

Peer) reviewing PDF | (2018:03:26444:1:1:NEW' 1 Jun 2018)
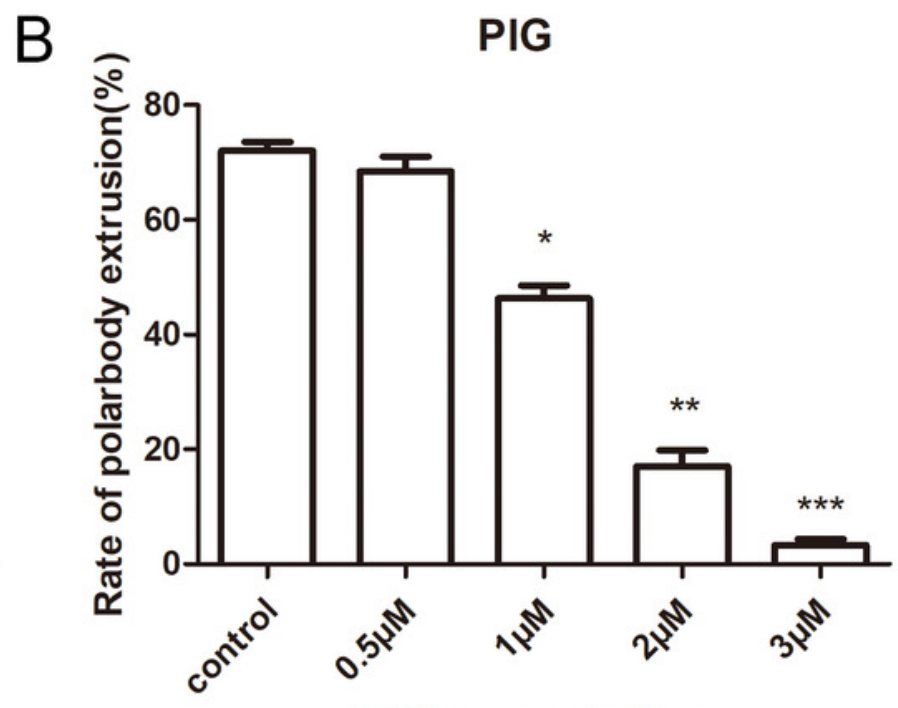

DON concentration

$\rightarrow$ MOUSE

$\rightarrow$ PIG 


\section{Figure 3}

The effects of HT-2 on the oocyte maturation

(A) Rate of polar body extrusion after HT-2 treatment. Mouse oocytes were cultured with HT2 at the concentration of $0,10,20,30,40 \mathrm{nM}$. (B) Rate of polar body extrusion after HT-2 treatment. For porcine oocytes culture, HT-2 was 0, 10, 50, 100, 150, 200, $400 \mathrm{nM}$. The rate of polar body extrusion was decreased when the concentration of HT-2 was increasing. (C) The statistical analysis for the effects of same concentration on mouse and porcine oocytes. The grey line represented rate of mouse polar body extrusion. The black line represented rate of porcine polar body extrusion. *, significance, $p<0.05$; **, significance, $p<0.01$; ***, significance, $p<0.001$. 
A

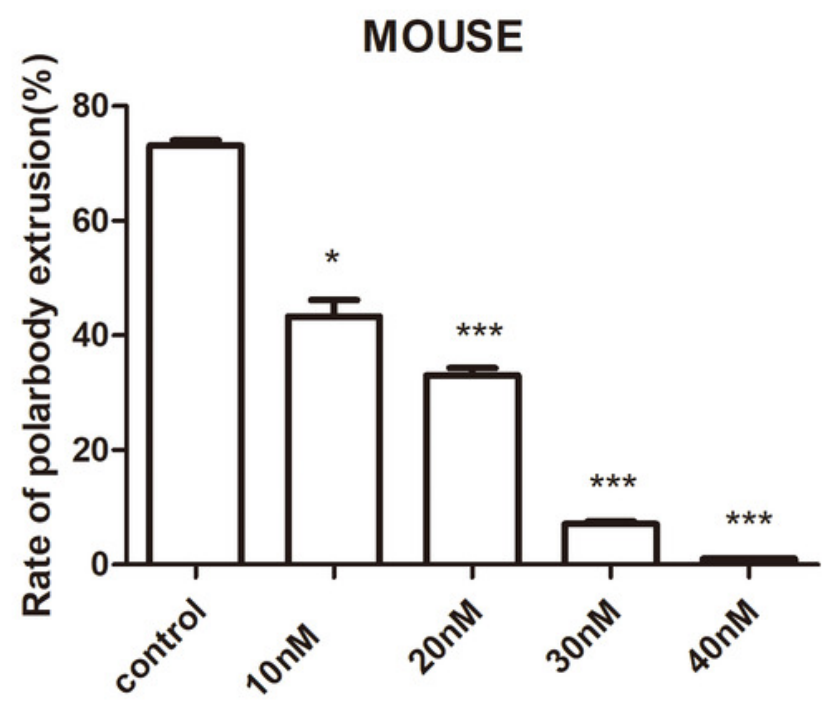

HT-2 concentration

C



HT-2 concentration/nM
$\mathrm{B}$

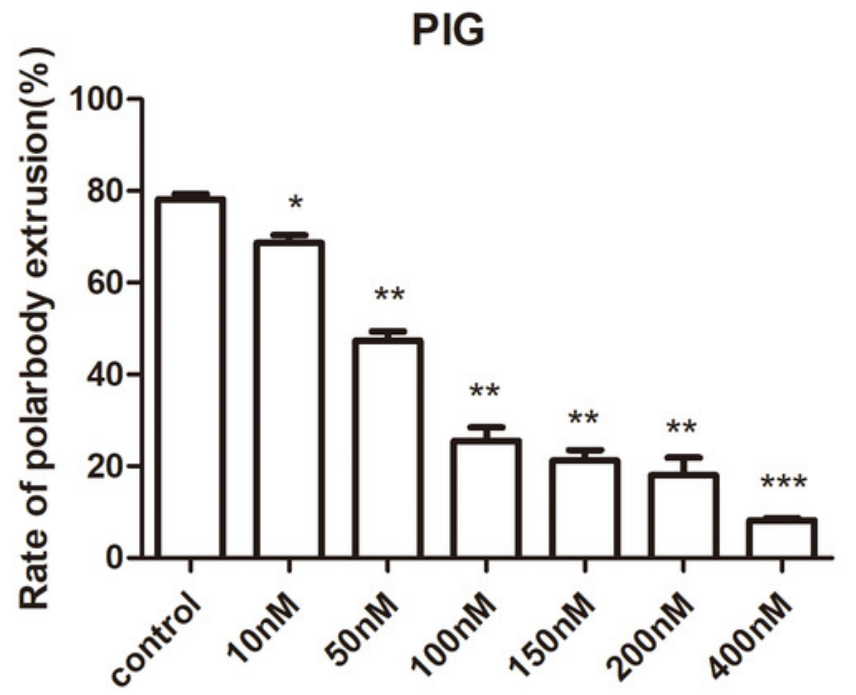

HT-2 concentration 


\section{Figure 4}

The effects of OTA on the oocyte maturation

(A) Rate of polar body extrusion after OTA treatment. For mouse oocyte culture, OTA concentration was $0,200,300,400,600 \mu \mathrm{M}$. (B) Rate of polar body extrusion after OTA treatment. For porcine oocyte culture, OTA concentration was $0,1,5,10,30,100 \mu \mathrm{M} . *$, significance, $p<0.05$; ${ }^{*}$, significance, $p<0.01$. (C) The comparison for the effects of different concentration on mouse and porcine oocytes. The grey line represented rate of mouse polar body extrusion. The black line represented rate of porcine polar body extrusion.

A

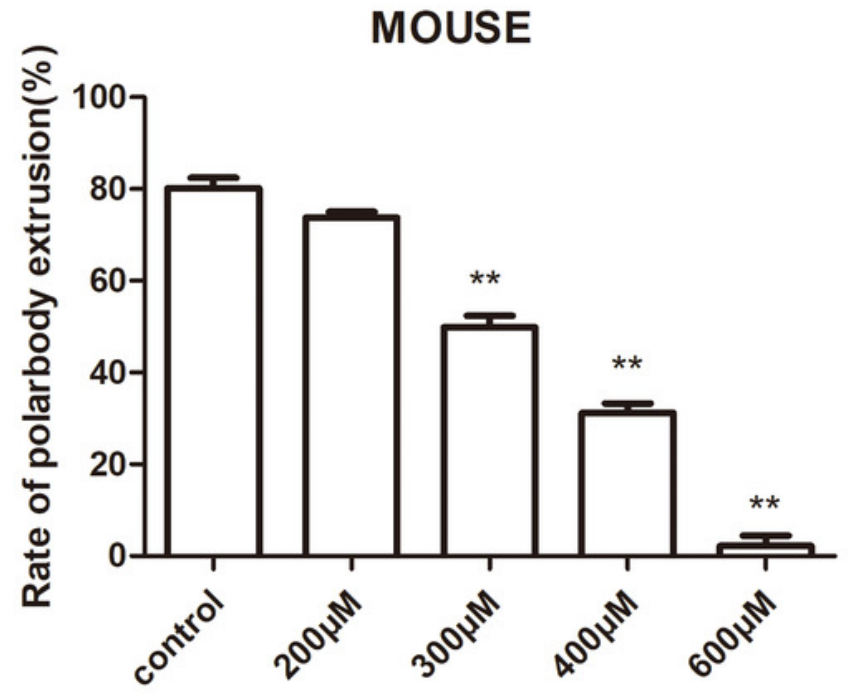

OTA concentration

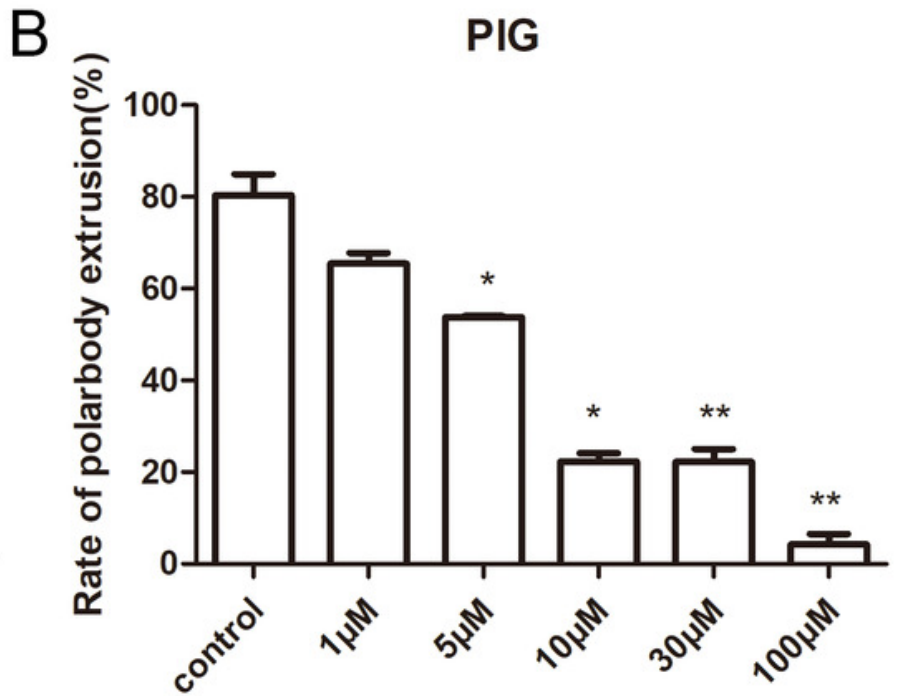

OTA concentration

C

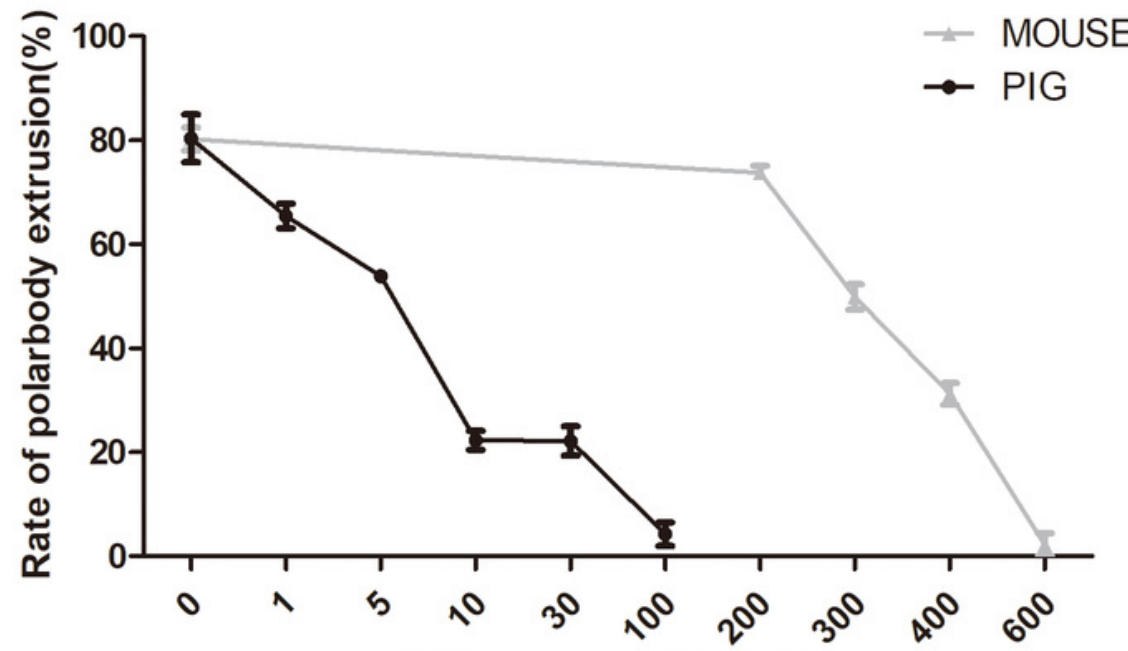




\section{Figure 5}

The effects of ZEA on the oocyte maturation.

(A) Rate of polar body extrusion after ZEA treatment. Mouse oocytes were cultured with 0 , 10, 50, 100, $200 \mu M$ ZEA. (B) Rate of polar body extrusion after ZEA treatment. Porcine oocytes were cultured with $0,5,10,30,50 \mu \mathrm{M}$ ZEA. Both mouse and porcine polar body extrusion rate were reducing when ZEA concentration was increasing. (C) The statistical analysis for the effects of same concentration on mouse and porcine oocytes. The grey line represented rate of mouse polar body extrusion. The black line represented rate of porcine polar body extrusion. *, significance, $p<0.05 ; * *$, significance, $p<0.01$. 
A

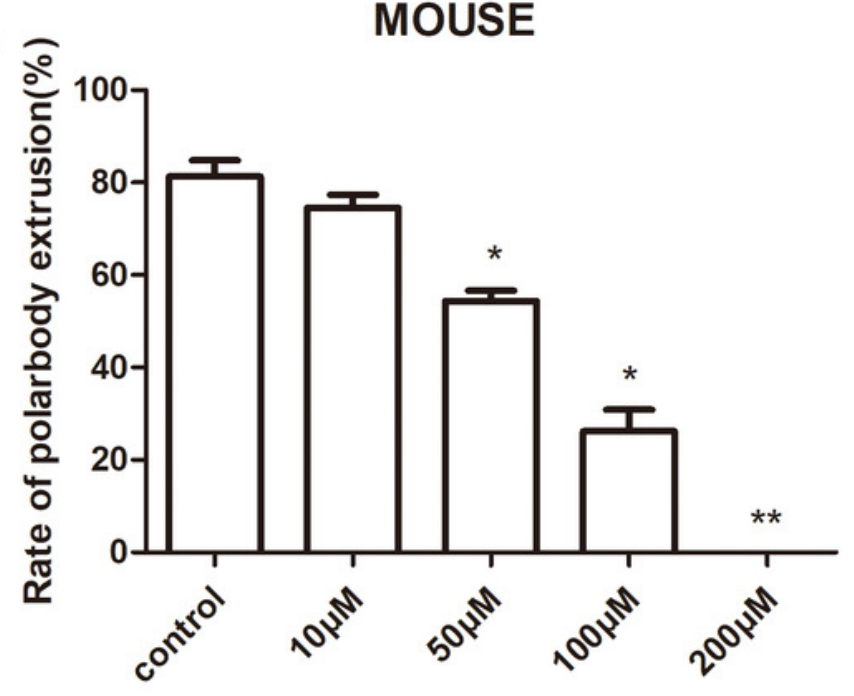

ZEA concentration

C

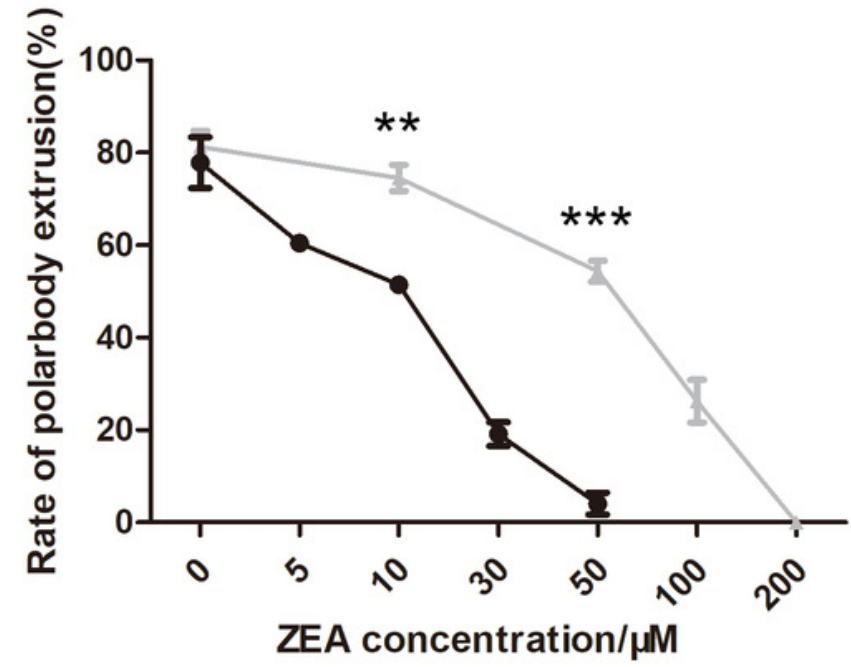

B

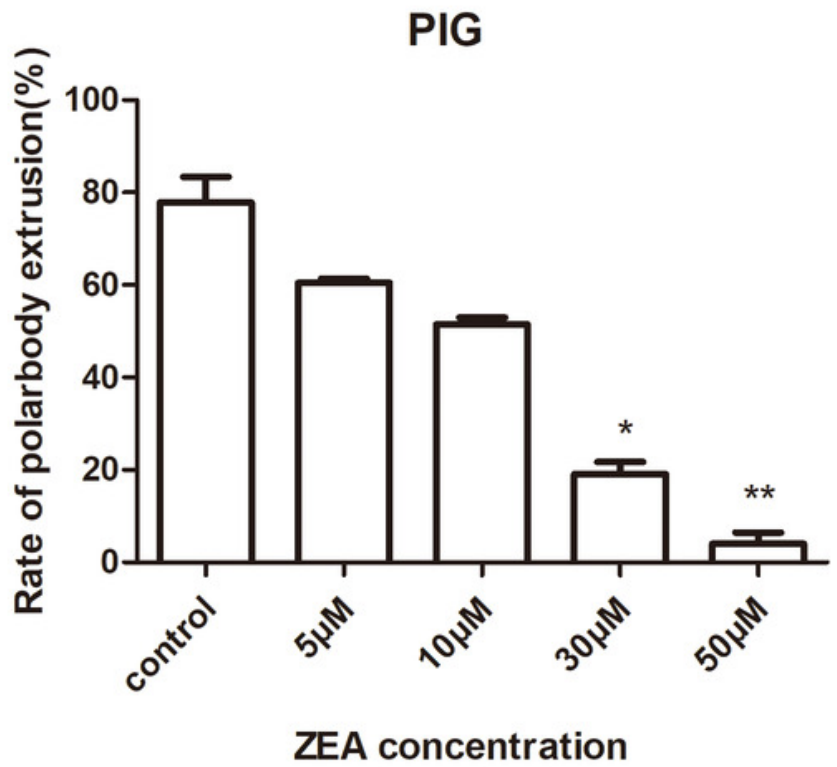

$\rightarrow$ MOUSE

$\rightarrow$ PIG 